Ann. Biol. anim. Bioch. Biophys., I966, 6 (3), 30I-3г3

\title{
MODIFICATION DE LA SPERMATOGENÈSE CHEZ LE CAMPAGNOL DES CHAMPS (MICROTUS ARVALIS) EN FONCTION DE LA DUREE QUOTIDIENNE D'ÉCLAIREMENT
}

\author{
Lise MARTINET \\ avec la collaboration technique de Monique Meunier \\ Station centrale de Physiologie animale, \\ Centre national de Recherches zootechniques, 78 - Jouy-en-Josas
}

\section{SOMMAIRE}

Deux expériences ont été réalisées, une sur le jeune entre o et 45 jours, l'autre chez l'adulte entre 7o et I 5 jours, pour connaître le rôle de durées quotidiennes d'éclairement croissantes ou décroissantes de $5 \mathrm{~h}$ en 45 jours.

Chez le jeune, des photopériodes claires croissantes ou décroissantes entre $5 \mathrm{~h}$ et io $\mathrm{h}$, to $\mathrm{h}$ et $\mathrm{I} 5 \mathrm{~h}$ ou $\mathrm{I} 5 \mathrm{~h}$ et $20 \mathrm{~h}$, permettent un développement de la spermatogenèse identique à celui provoqué par des photopériodes claires constantes de $5 \mathrm{~h}$, Io $\mathrm{h}, \mathrm{I} 5 \mathrm{~h}$ ou $20 \mathrm{~h}$ (MARTINET, I963).

Chez l'adulte, ayant reçu auparavant des éclairements quotidiens de $5 \mathrm{~h}, 10 \mathrm{~h}, \mathrm{I} 5 \mathrm{~h}$ ou $20 \mathrm{~h}$ par $24 \mathrm{~h}$, l'augmentation et la diminution de la photopériode claire ont mis en évidence une durée optimum d'éclairement situé entre $\mathrm{I}_{5} \mathrm{~h}$ et $20 \mathrm{~h}$. Quelle que soit la durée d'éclairement et le sens de la variation de cette durée, la spermatogenèse est maintenue.

\section{INTRODUCTION}

Si la durée quotidienne d'éclairement peut modifier l'établissement de la spermatogenèse chez le Campagnol, il semble qu'elle ne soit pas le seul facteur responsable des arrêts de reproduction observés dans la nature (LEcyk, I962; PINTER et NEGUS, I965).

Un travail précédent (MARTINET, I963) a montré que des durées quotidiennes courtes n'empêchaient pas l'établissement de la spermatogenèse chez le jeune, malgré une diminution de l'activité spermatogénétique; mais, les photopériodes claires 
utilisées étant de durée constante au cours de l'expérience, on pouvait objecter qu'elles ne correspondaient pas à la variation continuelle de la durée d'éclairement au cours du rythme naturel. D'autre part, une modification du régime lumineux $a-t$-elle une influence, la spermatogenèse une fois établie.

C'est pourquoi des animaux ont été soumis à des photopériodes claires croissantes ou décroissantes dans une première expérience pendant la période d'établissement de la spermatogenèse (o à 45 jours) ct dans une deuxième expérience, une fois la spermatogenèse établie (70 à II 5 jours).

\section{MA'TÉRIEL, E'T TECHNIQUES}

\section{Expérience $I$}

A partir de jeunes Campagnols nés de parents ayant subi auparavant des éclairements journaliers de $\mathrm{I} 5 \mathrm{~h}$, six lots ont été constitués et soumis à des photopériodes quotidiennes claires croissantes de $5 \mathrm{~h}$ à Io h (lot I), Io hà I $5 \mathrm{~h}$ (lot 2 ) et $15 \mathrm{~h}$ à $20 \mathrm{~h}$ (lot 3 ) ou décroissantes de $20 \mathrm{~h}$ à I $5 \mathrm{~h}$ (lot 4), I 5 h à Io h (lot 5) et io h à 5 h (lot 6). Ia durée quotidienne d'éclairement a augmenté ou diminué de 20 minutes tous les 3 jours, soit $5 \mathrm{~h}$ en 45 jours. après.

Les animaux étaient mis en expériences le jour de leur naissance et abattus 10, 20, 35 et 45 jours

\section{Expérience 11}

4 groupes d'animaux ont reçu des éclairements quotidiens établis suivant le plan ci-dessous :

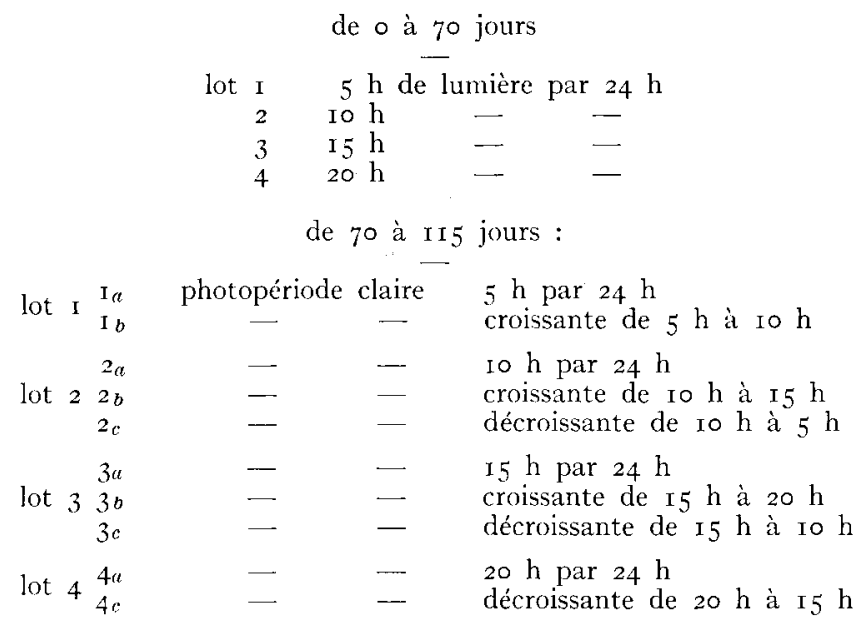

Tous les animaux ont été abattus à I I 5 jours.

Les techniques utilisées pour ces deux expériences sont celles décrites dans un premier article (Martinet, 1963 ).

L'étude quantitative de l'épithélium séminifère a été faite à un stade correspondant au stade I d'Ortavant (1958).

Le nombre total d'une catégorie de cellules germinales présentes dans le testicule, a été déterminé à partir d'une formule modifiée de celle d'Amann (MARTINET, I963).

Dans les différents lots, la comparaison de la croissance pondérale des testicules et des vésicules séminales a été réalisée par des analyses de covariance. 


\section{RÉSULTA'TS}

\section{Expérience $I$}

a) Croissance des testicules et des vésicules séminales (tabl. $x$, fig. I et 2 ).

Si l'on compare la croissance testiculaire des différents lots en tenant compte de la quantité totale de lumière reçue, on voit que jusqu'à 20 jours, il n'y a pas de différence entre les lots.

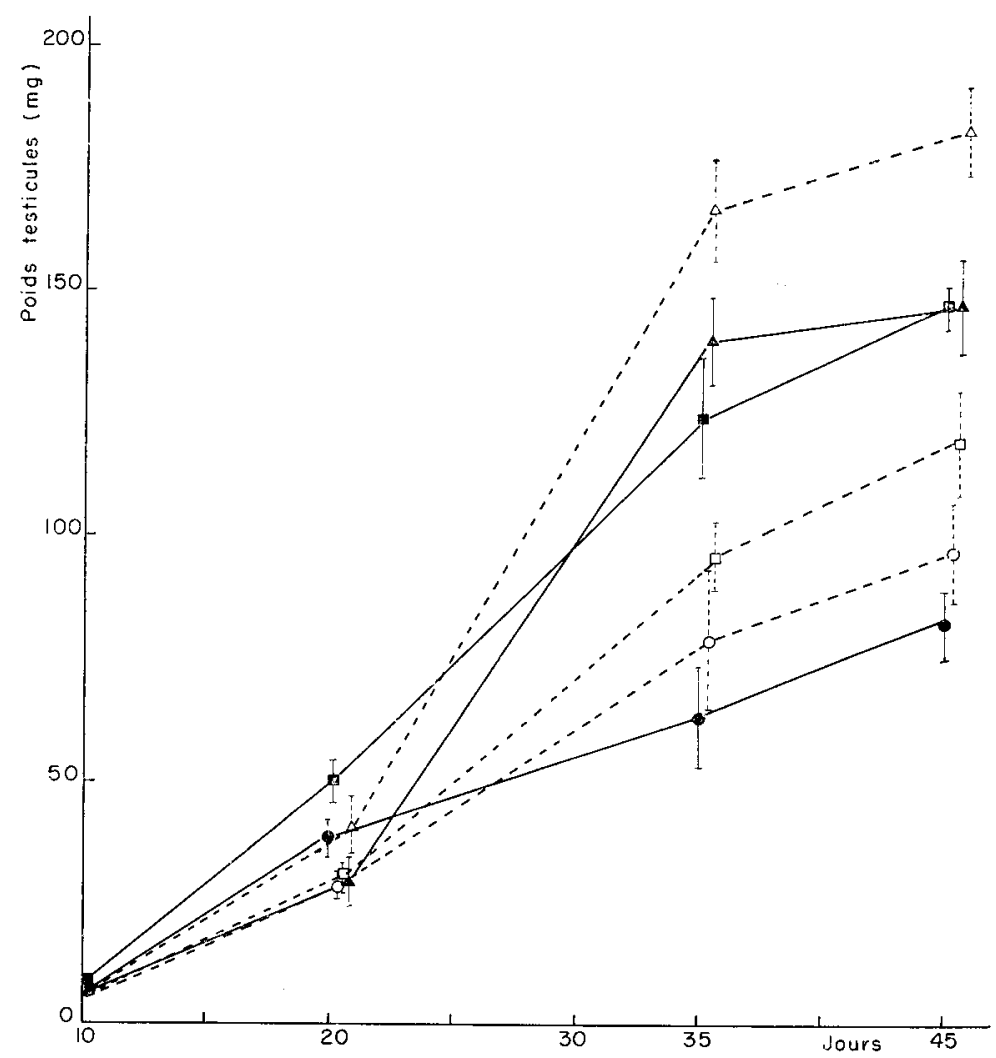

FIG I. - Croissance pondérale des lesticules en fonction de la photopériode

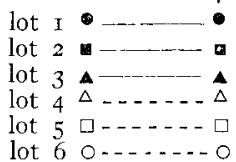

A 35 jours existe une corrélation linéaire significative entre la quantité totale de lumière reçue et le poids testiculaire, quel que soit le sens de la variation d'éclairement.

Entre 35 et 45 jours, à l'action de la quantité totale de lumière reçue s'ajoute l'effet provoqué par le sens de la variation d'éclairement; en effet, bien que la quan- 
TABLEAU I

Croissance pondérale des testicules et des vésicules séminales en fonction de la photopériode

\begin{tabular}{|c|c|c|c|c|c|c|c|}
\hline $\begin{array}{c}\text { Age } \\
\text { en jours }\end{array}$ & Lot & $\begin{array}{c}\text { Photopériode claire } \\
\text { (en heures) } \\
\text { passant de }\end{array}$ & $\begin{array}{c}\text { Quantité totale } \\
\text { de lumire } \\
\text { reçue } \\
\text { (en heures) }\end{array}$ & $\begin{array}{l}\text { Nbre } \\
\text { d'ani- } \\
\text { maux }\end{array}$ & $\begin{array}{c}\text { Poids } \\
\text { du corps } \\
(\mathrm{g} \pm \mathrm{sm})\end{array}$ & $\begin{array}{c}\text { Poids } \\
\text { des testicules } \\
(\mathrm{mg} \pm \mathrm{sm}) \\
\text { (moyenne) }\end{array}$ & $\begin{array}{c}\text { Poids } \\
\text { des vésicules } \\
\text { séminales } \\
(\mathrm{mg} \pm \mathrm{sm})\end{array}$ \\
\hline 10 & 1 & 5 à 10 par 24 & 54 & 6 & $5,3 \pm 0,1$ & 6,1 土 1,0 & \\
\hline 10 & 2 & 10 à $15-$ & 104 & 5 & $7,2 \pm 0,4$ & $9,0 \pm$ & \\
\hline 10 & 3 & 15 à 20 & 154 & 10 & $5,1 \pm 0,2$ & $6,4 \pm$ & \\
\hline 10 & 4 & 20 à 15 & 196 & 5 & 7,4 上 0,2 & $9,9 \pm$ & \\
\hline 10 & 5 & 15 à $10-$ & 146 & 10 & $6,0 \pm 0,4$ & $6,5 \pm$ & \\
\hline 10 & 6 & 10 à 5 & 96 & 5 & $5,8 \pm 0,2$ & $5,5 \pm 0,4$ & \\
\hline 20 & 1 & 5 à 10 par 24 & 122 & 10 & $13,9 \pm 0,6$ & $38,5 \pm 3,7$ & \\
\hline 20 & 2 & 10 à 15 & 222 & 10 & $16,4 \pm 1,6$ & $50,9 \pm$ & \\
\hline 20 & 3 & 15 à 20 & 322 & 10 & $10,9 \pm 0,7$ & $29,2 \pm$ & \\
\hline 20 & 4 & 20 à 15 & 378 & 10 & $12,0 \pm 0,6$ & $40,7 \pm$ & \\
\hline 20 & 5 & 15 à 10 & 278 & 10 & $10,9 \pm 0,5$ & $30,6 \pm$ & \\
\hline 20 & 6 & 10 à 5 & 178 & 10 & $13,2 \pm 0,4$ & $28,6 \pm$ & \\
\hline 35 & 1 & 5 à 10 par 24 & 238 & 15 & $17,8 \pm 0,8$ & $63,0 \pm 10,4$ & $41,2 \pm 13,1$ \\
\hline 35 & 2 & 10 à 15 & 413 & 15 & $22,7 \pm 1,4$ & $124,5 \pm 12,6$ & $103,6 \pm 17,9$ \\
\hline 35 & 3 & 15 à 20 & 588 & 17 & $15,9 \pm 0,8$ & $140,0 \pm 8,9$ & $57,1 \pm 12,6$ \\
\hline 35 & 4 & 20 à 15 & 633 & 15 & $18,8 \pm 1,1$ & $167,3 \pm$ & $141,3 \pm 15,7$ \\
\hline 35 & 5 & 15 à 10 & 458 & 14 & $18,5 \pm 0,8$ & $96,3 \pm 7,4$ & $50,9 \pm 8,0$ \\
\hline 35 & 6 & 10 à 5 & 283 & 15 & $21,3 \pm 1,0$ & $78,9 \pm 13,6$ & 67,7 上 19,8 \\
\hline 45 & 1 & 5 à 10 par 24 & 337 & 14 & 17,0 上. 0,6 & $82,5 \pm$ & $52,0 \pm 8,6$ \\
\hline 45 & 2 & 10 à 15 & 562 & 15 & $23,1 \pm 1,2$ & $148,6 \pm$ & $146,6 \pm 23,5$ \\
\hline 45 & 3 & 15 à 20 & 787 & 20 & $19,6 \pm 0,6$ & $147,7 \pm$ & $130,1 \pm 21,8$ \\
\hline 45 & 4 & 20 à 15 & 787 & 15 & $20,4 \pm 1,8$ & $183,0 \pm 8,8$ & $209,9 \pm 18,1$ \\
\hline 45 & 5 & 15 à 10 & 562 & 11 & $21,5 \pm 1,3$ & $119,8 \pm 10,6$ & $105,7 \pm 17,2$ \\
\hline 45 & 6 & 10 à 5 & 337 & 15 & $22,7 \pm 0,8$ & $97,1 \pm 10,6 \mid$ & $112,3 \pm 16,9$ \\
\hline
\end{tabular}

TABLEAU 2

Évolution des réserves épididymaires des Campagnols soumis à différentes photopériodes

\begin{tabular}{|c|c|c|c|c|c|}
\hline $\begin{array}{l}\text { Age } \\
\text { en jours }\end{array}$ & Lot & $\begin{array}{c}\text { Photopériode claire } \\
\text { passant de (en heures) }\end{array}$ & $\begin{array}{c}\text { Nombre } \\
\text { d'animaux }\end{array}$ & $\begin{array}{l}\text { Animaux } \\
\text { possédant } \\
\text { des réserves } \\
\text { épididymaires } \\
\text { (en p. 100) }\end{array}$ & $\begin{array}{c}\text { Réserves } \\
\text { épididymaires totales } \\
\text { chez les animaux } \\
\text { possédant des réserves } \\
\text { (moyenne) }\end{array}$ \\
\hline 35 & $\begin{array}{l}1 \\
2 \\
3 \\
4 \\
5 \\
6\end{array}$ & 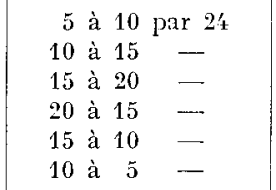 & $\begin{array}{l}15 \\
15 \\
17 \\
15 \\
14 \\
15\end{array}$ & $\begin{array}{r}40 \\
80 \\
88 \\
100 \\
64 \\
27\end{array}$ & $\begin{array}{l}5831 \times 10^{3} \\
6131 \times 10^{3} \\
4423 \times 10^{3} \\
5736 \times 10^{3} \\
3275 \times 10^{3} \\
3422 \times 10^{3}\end{array}$ \\
\hline 45 & $\begin{array}{l}1 \\
2 \\
3 \\
4 \\
5 \\
6\end{array}$ & $\begin{array}{rrrr}5 & \text { à } & 10 & \text { par } 24 \\
10 & \text { à } & 15 & - \\
15 & \text { à } & 20 & - \\
20 & \text { à } & 15 & - \\
15 & \text { à } & 10 & - \\
10 & \text { à } & 5 & \end{array}$ & $\begin{array}{l}14 \\
15 \\
20 \\
15 \\
11 \\
15\end{array}$ & $\begin{array}{r}6 / \mathbf{t} \\
100 \\
93 \\
100 \\
90 \\
73\end{array}$ & $\begin{array}{r}5955 \times 10^{3} \\
2810^{4} \times 10^{3} \\
42319 \times 10^{3} \\
63039 \times 10^{3} \\
10478 \times 10^{3} \\
16829 \times 10^{3}\end{array}$ \\
\hline
\end{tabular}


tité de lumière reçue soit la même pour les lots 2 et 5, d'une part, 3 et 4 d'autre part, la croissance testiculaire est la plus rapide dans les lots où la photopériode claire tend vers 15 heures.

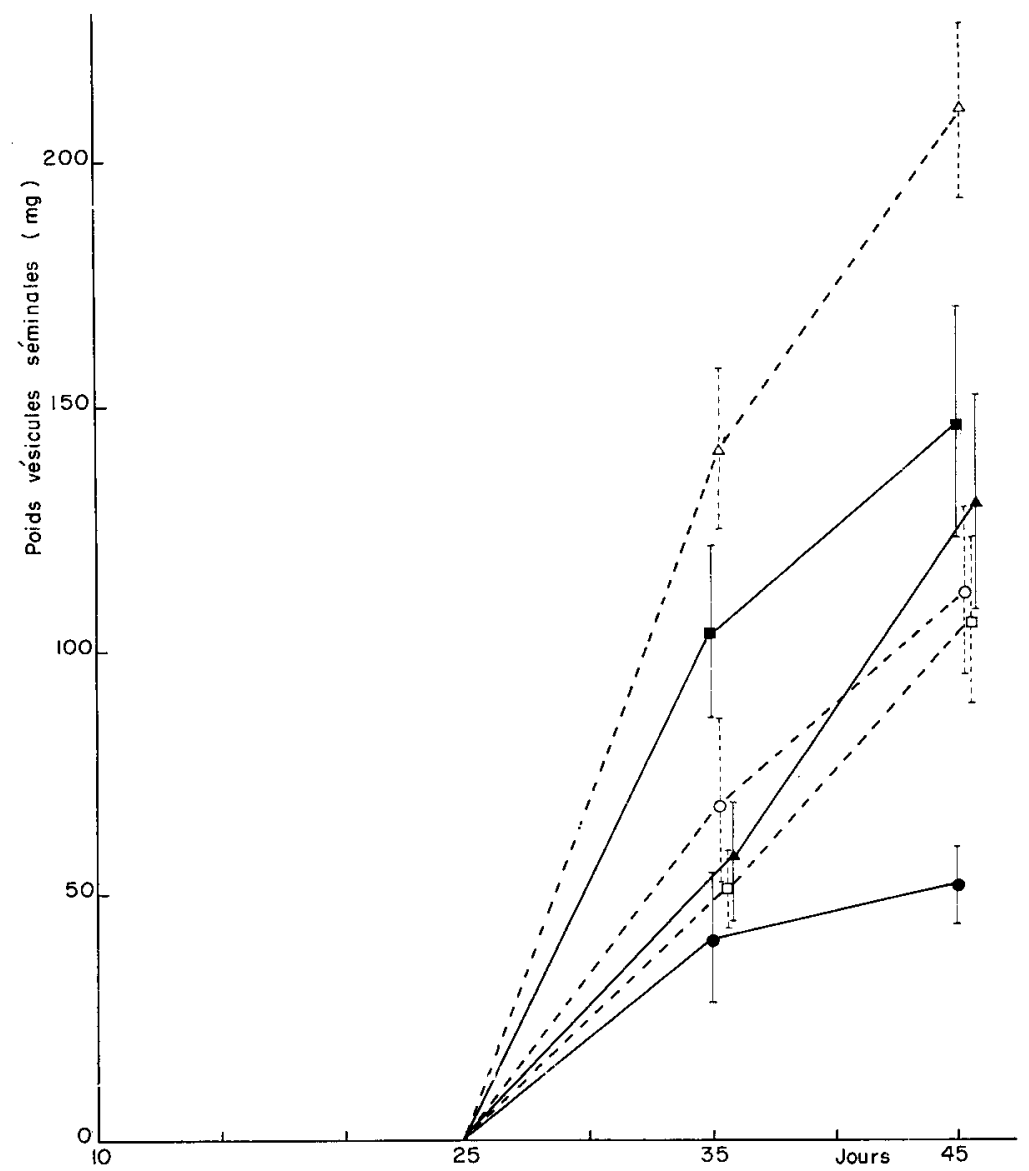

FIG. 2. - Croissance pondérale des vésicules séminales en fonction de la photopériode

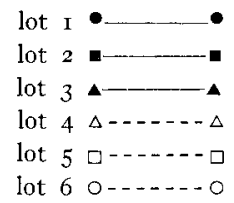

b) Réserves spermatiques épididymaires (tabl. 2).

Le pourcentage d'animaux possédant des réserves spermatiques épididymaires augmente en fonction de la quantité de lumière totale reçue pendant l'expérience.

Les réserves spermatiques épididymaires montrent les mêmes variations que celles observées pour la croissance testiculaire à savoir : augmentation des réserves avec l'augmentation des durées d'éclairement et pour des quantités égales de lu- 
nière reçue, réserves supérieures pour les photopériodes claires croissantes entre les niveaux Io heures et 15 heures et pour les photopériodes claires décroissantes entre les niveaux $\mathrm{r} 5$ heures et 20 heures.

c) Analyse histologique (tab1. 3).

L'étude quantitative des différentes catégories de cellules de l'épithélium séminifère permet de préciser les résultats obtenus avec les poids des testicules et les réserves spermatiques épididymaires.

TABLEAU 3

Étude histologique quantitative des testicules en fonction de la photopériode

\begin{tabular}{|c|c|c|c|c|c|c|c|c|c|}
\hline \multirow{2}{*}{$\begin{array}{c}\text { Age } \\
\text { (jours) }\end{array}$} & \multirow{2}{*}{ Lot } & \multirow{2}{*}{$\begin{array}{l}\text { Photo- } \\
\text { période } \\
\text { claire } \\
\text { (en heures) } \\
\text { passant de }\end{array}$} & \multirow{2}{*}{$\begin{array}{l}\text { Nbre } \\
\text { d'ani- } \\
\text { maux }\end{array}$} & \multicolumn{6}{|c|}{$\begin{array}{c}\text { Nombre de noyaux cellulaires par section transversale } \\
\text { de tube séminifère (nombre corrigé) }\end{array}$} \\
\hline & & & & Sertoli & $\begin{array}{l}\text { Spermato- } \\
\text { gonies }\end{array}$ & Leptotène & Pachytène & Spermatides & $\begin{array}{l}\text { Dégé-- } \\
\text { néres- } \\
\text { cences }\end{array}$ \\
\hline \multirow{6}{*}{20} & 1 & $5 \grave{a} 10$ & 10 & $29,0+1,5$ & $2,1+0,2$ & $37,2+3,9$ & $12,6+2,2$ & & 0,23 \\
\hline & 2 & 10 à 15 & 10 & $25,5 \pm 0,6$ & $2,6+0,2$ & $40,7+1,8$ & $17,9+1,7$ & & 0,36 \\
\hline & 3 & 15 à 20 & 10 & $28,5 \pm 0,9$ & $2,3 \pm 0,3$ & $34,9 \pm 2,6$ & $\begin{array}{r}8,0 \pm 1,5 \\
\end{array}$ & & 0,14 \\
\hline & 4 & 20 à 15 & 10 & $30,8+1,4$ & $2,7+0,5$ & $42,3+0,3$ & $12,6 \pm 0,3$ & & 0,68 \\
\hline & 5 & $15 \dot{\mathrm{a}} 10$ & 10 & $28,2 \neq 0,8$ & $1,9 \pm 0,1$ & $32,2 \pm 5,9$ & $10,0=\mathbf{1}, \mathbf{1}$ & & 0,16 \\
\hline & 6 & $10 \stackrel{\mathrm{a}}{5}$ & 10 & $30,0 \pm 1,1$ & $1,9 \pm 0,2$ & $28,6 \pm 2,0$ & $9,4 \pm 1,1$ & & 0,21 \\
\hline \multirow{6}{*}{35} & 1 & 5 à 10 & 10 & $26,4 \pm 2,6$ & $2,2 \pm 0,2$ & $25,6 \pm 2,4$ & $17,3 \pm 3,8$ & $34,7 \pm 11,0$ & 0,05 \\
\hline & 2 & 10 à 15 & 10 & $19,4 \pm 0,7$ & $2, \gamma_{1} \pm 0,3$ & $34,2 \pm 1,2$ & $32,2 \pm 1,9$ & $80,1 \pm 9, y_{t}^{\prime}$ & 0,02 \\
\hline & 3 & 15 à 20 & 10 & $18,0 \pm 0,9$ & $2,7 \pm 0,2$ & $34,0 \pm 1,9$ & $29,5 \pm 2,1$ & $80,2 \pm 9,7$ & 0,19 \\
\hline & 4 & 20 à 15 & 10 & $18,1 \neq 0,6$ & $2,9 \pm 0,3$ & $38,6 \pm 2,9$ & $40,0 \pm 2,6$ & $98,1 \pm 3,2$ & 0,09 \\
\hline & 5 & 15 à 10 & 10 & $20,9 \pm 1,3$ & $2,7 \pm 0,1$ & $38,7 \pm 1,1$ & $32,4- \pm 2,1$ & $63,0 \pm 9,5$ & 0,21 \\
\hline & 6 & 10 à 5 & 10 & $26,5 \pm 5,5$ & $2,2 \pm 0,1$ & $30,6 \pm 2,6$ & $13,3 \pm 3,2$ & $33,0 \pm 8,3$ & 0,21 \\
\hline \multirow{6}{*}{45} & 1 & 5 à 10 & 10 & $21,6 \pm 2,8$ & $3,2 \pm 0,2$ & $27,1 \pm 1,9$ & $23,0 \pm 2,6$ & $56,6 \pm 10,9$ & 0,07 \\
\hline & 2 & 10 à 15 & 10 & $18,7 \pm 0,6$ & $3,3 \pm 0,2$ & $29,5 \pm 1,4$ & $31,6 \pm 1,3$ & $87,4 \pm 4,8$ & 0,03 \\
\hline & 3 & 15 à 20 & 10 & $18,6 \pm 0,8$ & $3,8 \pm 0,5$ & $32,9 \pm 2,3$ & $25,5 \pm 2,8$ & $92,1 \pm \quad 9,8$ & 0,11 \\
\hline & 4 & 20 à 15 & 10 & $17,9 \pm 0,3$ & $2,5 \pm 0,2$ & $37, \pm 1,1$ & $32,3 \pm 1,6$ & $130,4 \pm 4,3$ & 0,05 \\
\hline & 5 & 15 à 10 & 10 & $18,7 \pm 0,8$ & $2,6 \pm 0,2$ & $33,9 \pm 1,3$ & $27,1 \pm 2,4$ & $102,1 \pm 11,8$ & 0,11 \\
\hline & 6 & 10 à 5 & 10 & $2_{t}^{\prime}, 7 \pm 2,9$ & $2,9 \pm 0,5$ & $3 y^{\prime}+1,3$ & $22, y_{1}^{\prime} \pm 3,7$ & $55,2 \pm 13,2$ & 0,02 \\
\hline
\end{tabular}

L'action de la durée d'éclairement se manifeste au niveau des divisions spermatogoniales, de la prophase méiotique et de la formation des spermatides.

En effet, si à 20 jours, il n'existe pas encore de différence entre les lots, à 35 et 45 jours, on observe une modification du nombre des spermatocytes au stade leptotène et pachytène et des spermatides à noyau rond en fonction de la durée d'éclairement. Les variations observées sont les mêmes que pour les poids testiculaires et les réserves épididymaires.

Le nombre de noyaux pycnotiques par section transversale de tube séminifère ne semble pas augmenter quand la durée d'éclairement diminue. 


\section{Expérience II}

Les tableaux 4, 5, 6 et 7 concernent les résultats de cette expérience. Les graphiques 3 et 4 ont réuni les résultats concernant l'expérience de Ig63 et celle-ci.

a) Poids des testicules et des vésicules séminales. (tab1. 4 et fig. 3).

A II 5 jours, le poids des testicules et des vésicules séminales d'animaux soumis depuis leur naissance à des photopériodes claires constantes ( $\operatorname{lots} \mathrm{I}_{a}, 2 a, 3 a, 4 a$ est maximum pour un éclairement journalier de $I_{5} \mathrm{~h}$. Il existe une différence très significative ( $\mathrm{P}<0,0 \mathrm{I})$ entre $\mathrm{I}_{a}, 2_{a}$, d'une part et $3 a$, d'autre part et une différence significative $(\mathrm{P}<0,05)$ entre $3 a$ et $4 a$.

\section{TABLEAU 4}

Poids à 115 jours des testicules et des vésicules séminales de Campagnols soumis à différentes photopériodes

\begin{tabular}{|c|c|c|c|c|c|}
\hline Lot & $\begin{array}{l}\text { Durée quotidienne } \\
\text { d'ćclairement }\end{array}$ & $\begin{array}{l}\text { Nombre } \\
\text { d'animaux }\end{array}$ & $\begin{array}{l}\text { Poids du corps } \\
\qquad(g \pm \mathrm{sm})\end{array}$ & $\begin{array}{c}\text { P'oids } \\
\text { des testicules } \\
\text { (moyenne) } \\
(\mathrm{mg} \pm \mathrm{sm})\end{array}$ & $\begin{array}{c}\text { Poids } \\
\text { des vésicules } \\
\text { séminales } \\
(\mathrm{mg} \dot{\mathrm{sm}})\end{array}$ \\
\hline $1_{a}$ & $5 \mathrm{~h}$ & 12 & $27,8 \pm 1,3$ & 130,9 上 8,9 & $232,9 \pm 31,4$ \\
\hline $1_{b}$ & $\begin{array}{l}5 \mathrm{~h} \text { jusqu'à } 70 \mathrm{j} \\
5 \mathrm{~h} \text { オ } 10 \mathrm{~h} \text { jusqu'à } 115 \mathrm{j}\end{array}$ & 12 & $29,,^{\prime} \pm 1,2$ & $133,6 \pm 12,0$ & $270,6 \pm 23,2$ \\
\hline $2 a$ & $10 \mathrm{~h}$ & 7 & $27,3 \pm 3,0$ & $150,,^{\prime} \pm 15,2$ & $193,0 \pm 29,7$ \\
\hline $2_{b}$ & $\begin{array}{l}10 \mathrm{~h} \text { jusqu'à } 70 \mathrm{j} \\
10 \mathrm{~h} \not 15 \mathrm{~h} \text { jusqu'à } 115 \mathrm{j}\end{array}$ & 10 & $33, \pm \pm 1, ' t$ & $209,6^{* *} \pm 16,7$ & $388,0^{* *} \pm 49,4$ \\
\hline $2 c$ & $\begin{array}{l}10 \mathrm{~h} \text { jusqu'à } 70 \mathrm{j} \\
10 \mathrm{~h} \searrow 5 \mathrm{~h} \text { jusqu'à } 115 \mathrm{j}\end{array}$ & 10 & $25,8 \pm 1,5$ & $123,5 \pm 10,8$ & $228,8 \pm 32,9$ \\
\hline $3_{a}$ & $15 \mathrm{~h}$ & 9 & $35,5=1,8$ & $227,6 \pm 16,5$ & $452,5 \pm 34,8$ \\
\hline $3 b$ & $\begin{array}{l}15 \mathrm{~h} \text { jusqu'à } 70 \mathrm{j} \\
15 \mathrm{~h} \not \nearrow 20 \mathrm{~h} \text { jusqu'à } 115 \mathrm{j}\end{array}$ & 8 & $3^{\prime}, 1 \pm 1,7$ & $239,6 \pm 10,3$ & $428, x \pm 25,6$ \\
\hline $3_{c}$ & $\begin{array}{l}15 \mathrm{~h} \text { jusqu'à } 70 \mathrm{j} \\
15 \mathrm{~h} \searrow 10 \mathrm{~h} \text { jusqu'à } 115 \mathrm{j}\end{array}$ & 10 & $33,0 \pm 2,1$ & $163,6^{* *} \pm 11,2$ & $335,5^{*} \pm 31,1$ \\
\hline $4 a$ & $20 \mathrm{~h}$ & 11 & 29,9 t= 2,5 & $187,4=12,6$ & $223,5=22,7$ \\
\hline${ }^{4} c$ & $\begin{array}{l}20 \mathrm{~h} \text { jusqu'à } 70 \mathrm{j} \\
20 \mathrm{~h} \searrow 15 \mathrm{~h} \text { jusqu'à } 115 \mathrm{j}\end{array}$ & 11 & $32,4 \pm 1,8$ & $230,9^{* *} \pm 15,6$ & $351,2^{* *} \pm 37,7$ \\
\hline
\end{tabular}

Les analyses de covariance ont été faites à l'intérieur de chacun des 'f lots et les comparaisons sont toujours faites par rapport à la durée d'éclairement constante ( $5 \mathrm{~h}, 10 \mathrm{~h}, 15 \mathrm{~h}$ ou $20 \mathrm{~h}$ ).

$* \mathrm{P}<0,05$.

$* * \mathrm{P}<0,01$.

La réponse des testicules et vésictules séminales à des modifications à partir de 70 jours, de la durée de la photopériode claire, dépend de la durée de cette photopériode avant 70 jours. 
Si l'on considère les photopériodes claires variant entre les niveaux 5 heures et Io heures (lots $I_{a}, I_{b}, 2_{a}$ et $2_{c}$ ) une augmentation ou une diminution de la durée d'éclairement ne modifie pas le poids des testicules et des vésicules séminales.

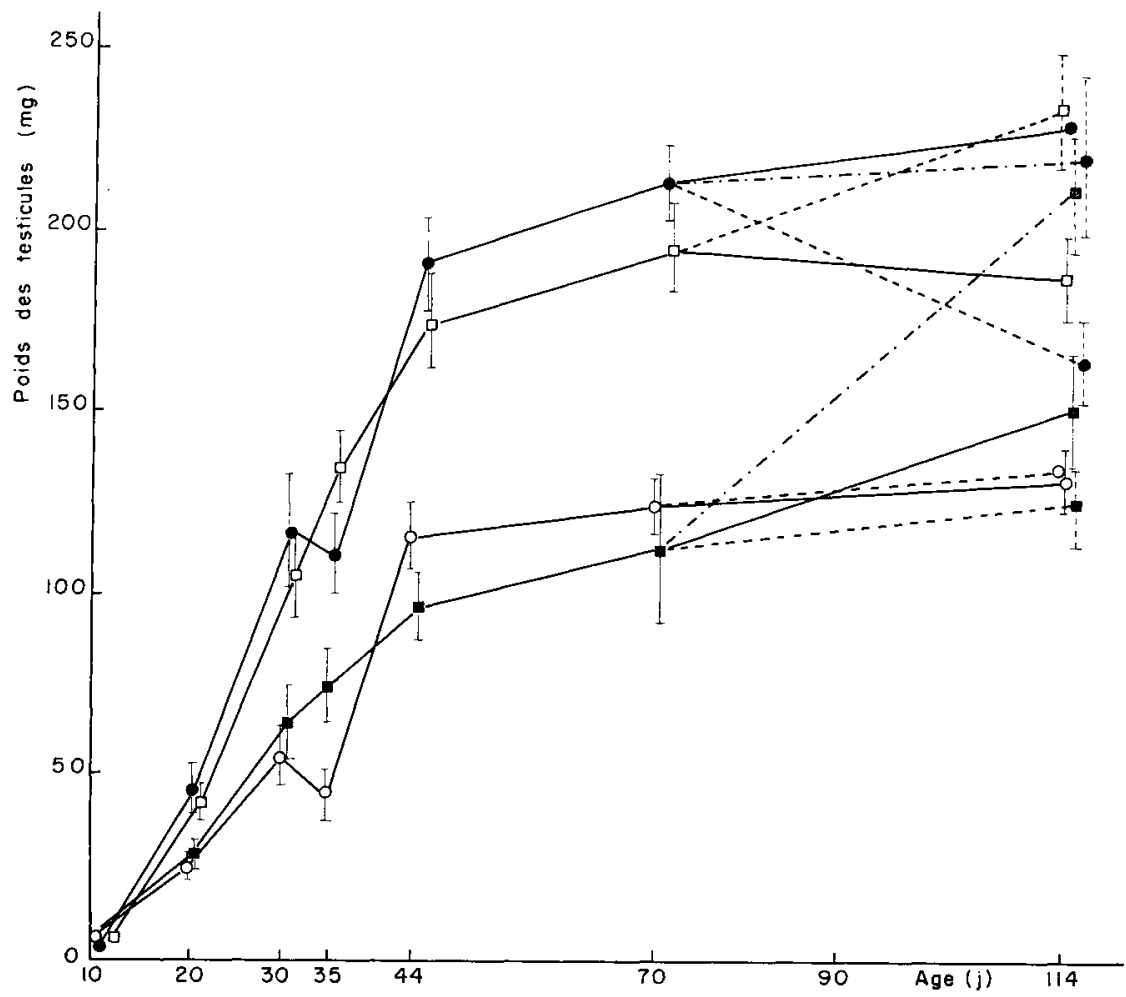

FIG. 3. - Evolution pondérale des testicules entre 0 et 115 jours, suivant différentes durées de photopériodes claires

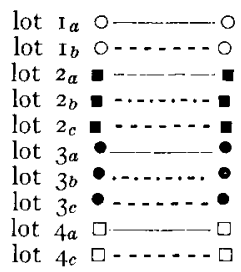

Au contraire, entre les niveaux Io heures et $I_{5}$ heures (lots $2_{a}, 2_{b}$ et $3 a, 3 c$ ), le passage de Io heures à $I_{5}$ heures d'éclairement quotidien provoque une augmentation très significative et, inversement, le passage de $\mathrm{r} 5$ heures à ro heures, une diminution très significative $d u$ poids des testicules et des vésicules séminales.

Entre les niveaux I 5 heures et 20 heures (lots $3 a, 3 b$, et $4 a, 4 c$ ), l'augmentation de 15 heures à 20 heures de lumière par 24 heures ne modifie pas le poids des testicules et des vésicules séminales, mais le passage de 20 heures à I 5 heures l'augmente significativement. 
I,e figure 3, réunissant les données d'une expérience précédente (MaRTINET, I963) et celles de l'expérience présente, permet de suivre l'évolution pondérale des testicules en fonction de la photopériode.

b) Réserves épididymaires. (tab1 5)

A II5 jours, tous les animaux, quelle que soit la durée de la photopériode, possèdent des réserves épididymaires en quantité suffisante pour permettre la fécondation.

Mais 1'importance de ces réserves subit des variations identiques à celles observées pour le poids des testicules.

\section{TABLEAU 5}

Réserves épididymaires à 115 jours des Campagnols soumis à différentes photopériodes

\begin{tabular}{|c|c|c|c|c|}
\hline Lot & $\begin{array}{l}\text { Durée quotidienne } \\
\text { d'éclairement }\end{array}$ & $\begin{array}{c}\text { Nombre } \\
\text { d'animaux }\end{array}$ & $\begin{array}{l}\text { Animaux possédant } \\
\text { des réserves } \\
\text { épididymaires } \\
\text { (en p. 100) }\end{array}$ & $\begin{array}{c}\text { Réserves } \\
\text { épididymaires totales } \\
\text { chez les animaux } \\
\text { possédant des réserves } \\
\text { (moyenne) }\end{array}$ \\
\hline $1_{a}$ & $5 \mathrm{~h}$ & 12 & 100 & $59373 \times 10^{3}$ \\
\hline $1_{b}$ & $\begin{array}{l}5 \mathrm{~h} \text { jusqu'à } 70 \mathrm{j} \\
5 \mathrm{~h} \nearrow 10 \mathrm{~h} \text { jusqu'à } 115 \mathrm{j}\end{array}$ & 12 & 100 & $38510 \times 10^{3}$ \\
\hline $2 a$ & $10 \mathrm{~h}$ & 7 & 100 & $61592 \times 10^{3}$ \\
\hline $2_{b}$ & $\begin{array}{l}10 \mathrm{~h} \text { jusqu'à } 70 \mathrm{j} \\
10 \mathrm{~h} \nearrow 15 \mathrm{~h} \text { jusqu'à } 115 \mathrm{j}\end{array}$ & 10 & 100 & $79534 \times 10^{3}$ \\
\hline $2 c$ & $\begin{array}{l}10 \mathrm{~h} \text { jusqu'à } 70 \mathrm{j} \\
10 \mathrm{~h} \searrow 5 \mathrm{~h} \text { jusqu’à } 115 \mathrm{j}\end{array}$ & 10 & 100 & $5078^{\prime} \mathrm{k} \times 10^{3}$ \\
\hline $3_{a}$ & $15 \mathrm{~h}$ & 9 & 100 & $113732 \times 10^{3}$ \\
\hline $3_{b}$ & $\begin{array}{l}15 \text { h jusqu'à } 70 \mathrm{j} \\
15 \mathrm{~h} \nearrow 20 \mathrm{~h} \text { jusqu'à } 115 \mathrm{j}\end{array}$ & 8 & 100 & $13540^{4} \times 10^{3}$ \\
\hline $3_{c}$ & $\begin{array}{l}15 \mathrm{~h} \text { jusqu’à } 70 \mathrm{j} \\
15 \mathrm{~h} \searrow 10 \mathrm{~h} \text { jusqu’à } 115 \mathrm{j}\end{array}$ & 10 & 100 & $67872 \times 10^{3}$ \\
\hline $4 a$ & $20 \mathrm{~h}$ & 11 & 100 & $92912 \times 10^{3}$ \\
\hline $\mathbf{1}_{c}$ & $\begin{array}{l}20 \text { h jusqu'à } 70 \mathrm{j} \\
20 \mathrm{~h} \searrow 15 \mathrm{~h} \text { jusqu'à } 115 \mathrm{j}\end{array}$ & 11 & 100 & $102606 \times 10^{3}$ \\
\hline
\end{tabular}

c) Étude quantitative de l'épithélium séminifère (tab1. 6 et 7).

Comme pour l'expérience I, les numérations ont été effectuées sur les sections transversales de tubes au stade $I$.

On constate une diminution du nombre total de chaque catégorie cellulaire à l'exclusion des spermatogonies quand on passe des lots à longue durée d'éclairement $(3 a, 4 a)$ aux lots à faible durée d'éclairement $\left(2_{a}, \mathrm{I}_{a}\right)$. 


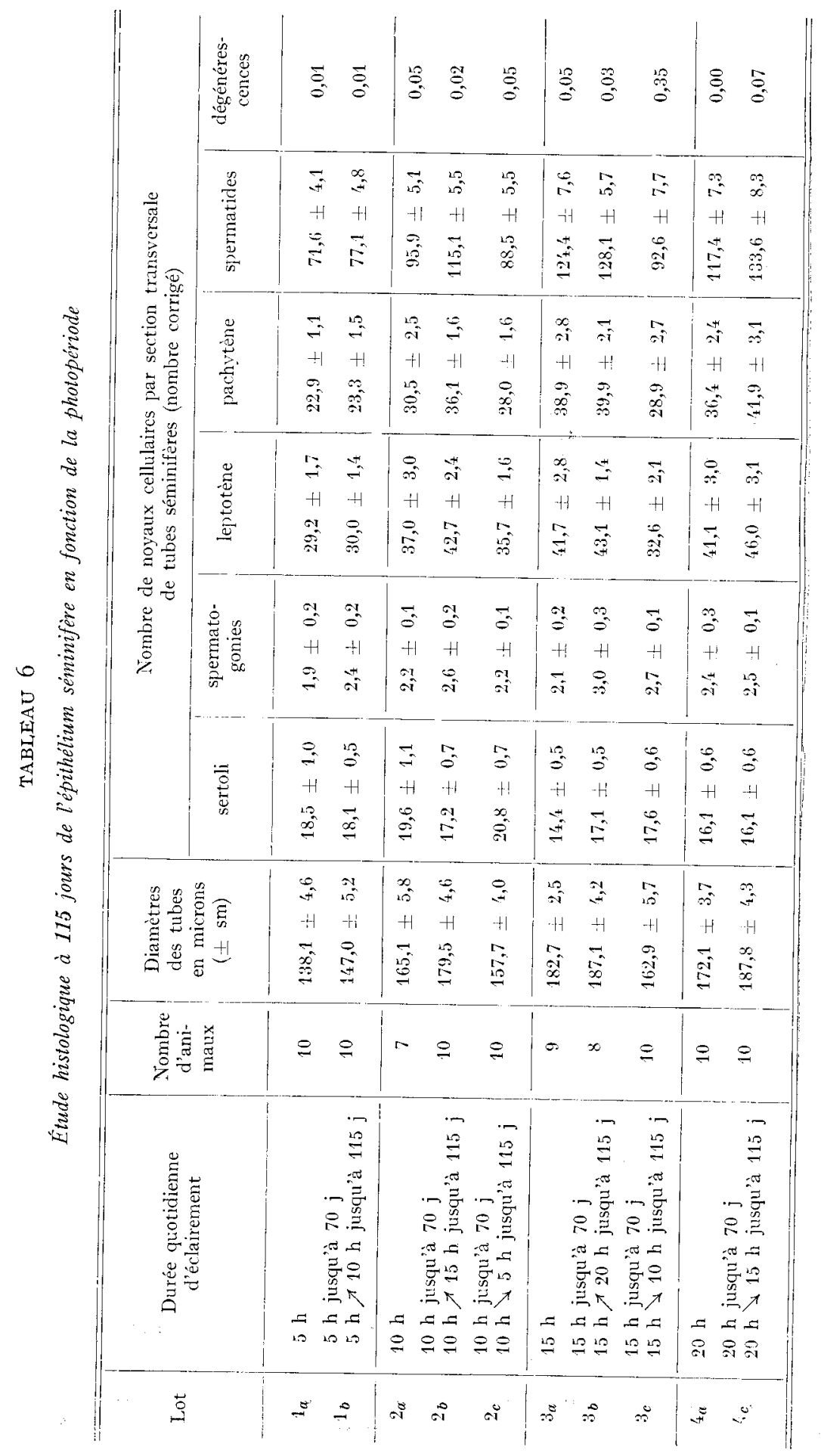


Chez les animaux ayant subi une modification du régime lumineux entre 70 et II 5 jours, le nombre de chaque catégorie cellulaire n'évolue pas pour les durées. d'éclairement quotidien comprises entre 5 heures et Io heures ( $\operatorname{lots} \mathrm{I}_{a}, \mathrm{I}_{b}, 2_{a}, 2_{c}$ ).

\section{TABI,EAU 7}

Nombre total à 115 jours de cellules germinales au stade leptotène de la prophase méïotique dans le testicule pour le stade étudié

(Ce nombre est porportionnel au nombre exact)

\begin{tabular}{|c|c|c|c|}
\hline I.ot & $\begin{array}{l}\text { Durée quoticlienne } \\
\text { cl'éclairement }\end{array}$ & $\begin{array}{l}\text { Nombre } \\
\text { d'animaux }\end{array}$ & $\begin{array}{l}\text { Nombre total } \\
\text { de leptotenes }\end{array}$ \\
\hline $1_{a}$ & $5 \mathrm{~h}$ & 10 & $685 \div 800$ \\
\hline $1_{b}$ & $\begin{array}{l}5 \text { h jusqu'à } 70 \mathrm{j} \\
5 \mathrm{~h} \not 10 \mathrm{~h} \text { jusqu'à } 115 \mathrm{j}\end{array}$ & 10 & 5697100 \\
\hline$\ddot{q}_{a}$ & $10 \mathrm{~h}$ & 7 & 6677800 \\
\hline $2 b$ & $\begin{array}{l}10 \mathrm{~h} \text { jusqu'à } 70 \mathrm{j} \\
10 \mathrm{~h} \not 15 \mathrm{~h} \text { jusqu'à } 115 \mathrm{j}\end{array}$ & 10) & 9072000 \\
\hline$\ddot{z}_{c}$ & $\begin{array}{l}10 \mathrm{~h} \text { jusqu'à } 70 \mathrm{j} \\
10 \mathrm{~h} \forall 5 \mathrm{~h} \text { jusqu'à } 115 \mathrm{j}\end{array}$ & 10 & 5735700 \\
\hline$z_{a}$ & $15 \mathrm{~h}$ & 9 & 9335000 \\
\hline $3_{b}$ & $\begin{array}{l}15 \mathrm{~h} \text { jusqu'à } 70 \mathrm{j} \\
15 \mathrm{~h} \nearrow 20 \mathrm{~h} \text { jusqu'à } 115 \mathrm{j}\end{array}$ & 8 & 9759900 \\
\hline $3 c$ & $\begin{array}{l}15 \text { h jusqu’à } 70 \mathrm{j} \\
15 \text { h } \ 10 \text { h jusqu’à } 115 \mathrm{j}\end{array}$ & 10 & 6515500 \\
\hline $4_{a}$ & $20 \mathrm{~h}$ & 10 & 8320400 \\
\hline$l_{t} c$ & $\begin{array}{l}20 \text { h jusqu'à } 70 \mathrm{j} \\
20 \text { h } \searrow 15 \text { h jusqu’a } 115 \mathrm{j}\end{array}$ & 10 & 9831600 \\
\hline
\end{tabular}

Pour les photopériodes claires comprises entre ro heures et I5 heures (lots $2_{a}$, $2_{b}$ et $3 a, 3 c$ ), le nombre de cellules germinales augmente quand la photopériode augmente et diminue quand la photopériode diminue.

Pour les photopériodes claires comprises entre I5 heures et 20 heures (lots $3 a$, $3 b$ et $4 a, 4$ ) les différences observées ne sont pas significatives.

Le nombre de noyaux en dégénérescence est identique dans tous les lots sauf le lot $3 c$ où la durée quotidienne d'éclairement passe de $I_{5}$ heures à Io heures par 24 heures : le nombre de dégénérescences augmente de manière très appréciable.

Le nombre total de cellules germinales au stade leptotène de la prophase méiotique a été calculé (tabl. 7). Le nombre est plus grand pour les photopériodes claires longues, I 5 heures et 20 heures (lots $3 a, 4 a$ ) que pour les photopériodes claires courtes, Io heures et 5 heures (lots $\mathrm{I}_{a}$, et $2_{a}$ ). 
La figure 4 permet de suivre l'évolution du nombre de cellules germinales au stade leptotène en fonction de la photopériode. On peut remarquer que cette évolution est tout à fait parallèle à celle du poids testiculaire.

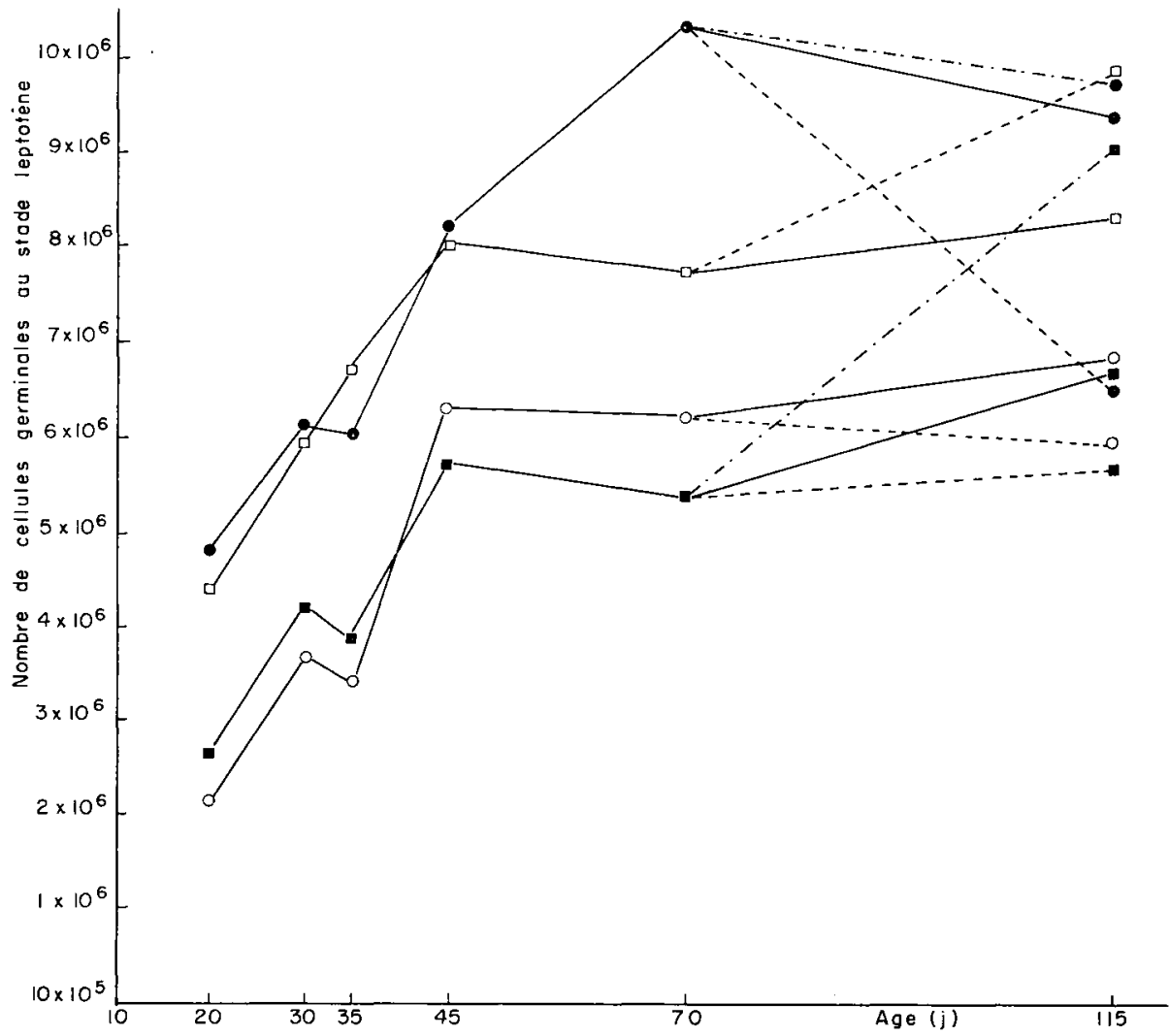

FIG. 4. - Evolution du nombre total de cellules germinales au stade Leptotène suivant différentes durées de photopériodes claires

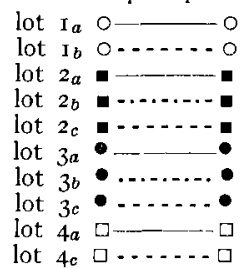

\section{DISCUSSION}

De la première expérience, on peut conclure que :

- des photopériodes claires croissantes ou décroissantes ne provoquent pas une stimulation ou une inhibition de l'établissement de la spermatogenèse supérieures à celles provoquées par des photopériodes claires constantes ; 
- on retrouve les deux niveaux de croissance testiculaires : élevés pour les photopériodes claires comprises entre I5 heures et 20 heures, faibles pour celles comprises entre 5 heures et ro heures.

La deuxième expérience cherchait à montrer l'effet d'une modification de la durée de la photopériode sur la spermatogenèse des adultes.

Il semble qu'il y ait une durée optimum d'éclairement quotidien située entre I 5 heures et 20 heures; dès que l'on s'en éloigne, le rendement de la spermatogenèse diminue.

Le nombre de lignées germinales mises en route (mesuré par le nombre total, par testicule, de spermatocytes au stade leptotène) est plus faible pour les photopériodes claires courtes (5 heures et Io heures) que pour les longues (I5 heures et 20 heures).

Mais une augmentation de la photopériode claire permet la mise en route de nouvelles lignées germinales ; et, inversement, une diminution de la durée d'éclairement provoque une diminution du nombre de spermatogonies actives.

Il semble que le nombre de cellules souches ne soit pas diminué par des faibles durées d'éclairement puisqu'il suffit d'augmenter la durée d'éclairement pour provoquer un développement important du nombre de spermatocytes au stade leptotène.

En aucun cas, il n'a été possible de provoquer des régressions complètes de la spermatogenèse. Il faudrait donc chercher l'influence d'un autre facteur que celui de la photopériode pour expliquer, si elles existent, les régressions testiculaires de la fin de la saison de reproduction dans la nature.

\title{
Reçu pour publication en juin 1966.
}

\author{
SUMMARY \\ ALTERATIONS IN THE SPERMATOGENESIS OF THE FIELD VOLE \\ IN RELATION TO DAILY DURATION
}

Two investigations : one on adult field voles aged 70 to II 5 days; one on the young aged o to 45 days; were undertaken in order to determine the effect of increases or decreases ( 5 hours in 45 days) in daily illumination.

In the young, spermatogenesis increases identically both under daily photoperiods increasing or decreasing from 5 to 10 , IO to 15 or 15 to 20 hours and under constant illuminations of $5,10,15$ or 20 hours per day.

In the adult who had been previously submitted to a 5 , Iо, I 5 or 20 hour daily illumination, experimental increases and decreases revealed an optimum length between 15 and 20 hours. Spermatogenesis holds on however, whatever the length of daily illumination and the direction of its variation may be.

\section{RÉFÉRENCES BIBLIOGRAPHIQUES}

Lecrk M., Ig52. The effect of the length of day light on reproduction in the field vole (Microtus arvalis). Zool. Pol., 12, I89-221.

Martinet L., 1963. Établissement de la spermatogenèse chez le Campagnol des Champs (Microtus arvalis) en fonction de la durée quotidienne d'éclairement. Ann. Biol. anim. Bioch. Biophys., 3, 343-352.

Ortavant R., 1958. Le cycle spermatogénélique chez le Bélier. Thèse Fac. Sci., Paris.

Pinter A. J., Negus M. C., 1965. Effects of nutrition and photoperiod on reproductive physiology of Microtus inontanus. Amer. J. Physiol., 208, 633-638.

Annales de Biologie animale. - r966. 